\title{
Elevated Expression of Periostin in Cartilage in Early Experimental Osteoarthritis
}

\author{
Rongkai Zhang ${ }^{1, \dagger}$, Guowei Li ${ }^{1, \dagger}$, Dong Jiang ${ }^{2, \dagger}$, Lukun Yang $^{3}$, Dawei Zhang ${ }^{1}, Z_{\text {he Wang }}{ }^{1}$, \\ Jun Liang ${ }^{4}$, *
}

${ }^{1}$ Department of Orthopaedics, the Fifth Affiliated Hospital of Sun Yat-sen University, Zhuhai, China

${ }^{2}$ Department of Urology, the Fifth Affiliated Hospital of Sun Yat-sen University, Zhuhai, China

${ }^{3}$ Department of Anesthesiology, the Fifth Affiliated Hospital of Sun Yat-sen University, Zhuhai, China

${ }^{4}$ Department of Stomatology, the Fifth Affiliated Hospital of Sun Yat-sen University, Zhuhai, China

\section{Email address:}

kaican@163.com (Jun Liang)

${ }^{*}$ Corresponding author

$\dagger$ These authors contributed equally to this work.

\section{To cite this article:}

Rongkai Zhang, Guowei Li, Dong Jiang, Lukun Yang, Dawei Zhang, Zhe Wang, Jun Liang. Elevated Expression of Periostin in Cartilage in Early Experimental Osteoarthritis. Biomedical Sciences. Vol. 3, No. 6, 2017, pp. 114-118. doi: 10.11648/j.bs.20170306.12

Received: October 19, 2017; Accepted: November 1, 2017; Published: December 5, 2017

\begin{abstract}
Osteoarthritis (OA) is characterized by the degeneration of affected joints leading to pain and reduced mobility. Periostin appears as a potential candidate in transducing mechanical signals to stimulate chondrocyte proliferation. 15 rats (E-group) underwent both medial meniscectomy and medial collateral ligament (MCL) transaction, while the other 15 rats (S-group) underwent sham surgery. Animals were killed at 1,2, and 4 weeks postsurgery, and 5 animals were put into use per-time point in each treatment group. Cartilage was harvested from tibial plateau and femoral condyle. We examined the level of Postn expression in cartilage of the experimental OA, using real-time PCR and immunohistochemistry analysis. The expression of Postn was about 2 -fold higher at 1 week $(\mathrm{P}=0.08<0.05)$, 4-fold higher at 2 weeks $(\mathrm{P}=0.003<0.05)$ and 4 -fold higher at 4 weeks $(\mathrm{P}=0.034<0.05)$ post-surgery in $\mathrm{E}$-group compared to $\mathrm{S}$-group. Postn protein expression levels were markedly increased in the E-Group compared with the S-Group at 1,2 and 4 weeks post-surgery. This study will be of benefit to those subsequent studies using the OA model to evaluate the outcomes of Postn epxpression in mechanism of OA. Postn may play prominent roles in pathogenic mechanisms of OA.
\end{abstract}

Keywords: Osteoarthritis (OA), Periostin (Postn), Cartilage, Rat Model of Osteoarthritis

\section{Introduction}

Osteoarthritis (OA) is a common complex disease of high public health burden, characterized by the degeneration of affected joints leading to pain and reduced mobility. However, the molecular pathophysiological OA are complex and poorly understood [1].

Both normal physiologic and pathologic overload forces are known to regulate cellular responses in essentially all musculoskeletal connective tissues [2]. Many previous studies revealed that alter joint loading patterns could lead to OA progression [3,4]. Molecular changes in response to mechanical stimuli are likely etiologic for OA at early stages. Periostin (POSTN) is a vitamin K-dependent glutamate-containing matri-cellular protein, belonging to the fasciclin family. It was originally identified in mouse osteoblasts and promotes cell adhesion and migration [5]. It appears as a potential candidate in transducing mechanical signals to stimulate chondrocyte proliferation [6]. Indeed, our previous study in experimental rat OA model found that the POSTN gene is significantly upregulated in the subchondral bone at 1 week post-surgery. And we have speculated that Postn expression is stimulated by the abnormal mechanical force of the surgery in the subchondral bone, causing angiogenesis and metalloproteinase-induced cellular matrix degeneration, 
thus initiating cartilage loss [7]. We hypothesis that Postn expression is also upregulated in joint cartilage under pathologic overload forces. To test the hypothesis, the experimental rat OA model was used in this study, which knee joint surgical instability induced pathologic overload forces on cartilage [7]. The level of Postn expression was studied in cartilage of the experimental OA by real-time PCR and immunohistochemistry analysis.

In the current study, rat model of OA cartilage expressed high levels of Postn in a time-dependent manner with disease progression, suggesting a previously unrecognized role of Postn in the pathogenesis of very early stages of OA. Postn may be a potential therapeutic target for blocking the progress.

As we know, this work is the first study to analysis the time course of the expression changes of Postn in cartilage in the very early stages of rat experimental OA.

\section{Materials and Methods}

\subsection{Rat Model of Osteoarthritis Cartilage Procurement}

OA was surgically induced as described previously [7], 15 rats (experimental group, E-group) underwent open surgery, involved in both medial meniscectomy and medial collateral ligament (MCL) transaction, while the other 15 rats (sham-operated group, S-group) underwent sham surgery. Animals were killed at 1,2 , and 4 weeks postsurgery, and 5 animals were put into use per-time point in each treatment group. Cartilage was harvested from tibial plateau and femoral condyle in liquid nitrogen as described previously [7], and immersed immediately in Trizol reagent (Life Technologies) at $-4^{\circ} \mathrm{C}$, then stored at $-80^{\circ} \mathrm{C}$ until RNA extraction.

Every procedure was reviewed and approved by the Institutional Animal Care and Use Committee of the Fifth Affiliated Hospital of Sun Yat-sen University (Approval ID: 20151281).

\subsection{Real-Time Polymerase Chain Reaction (PCR) Analysis}

According to the manufacturer's instructions, total Ribonucleic acid (RNA) of the cartilage was extracted with the E. Z. N. A Total RNA Kit II (Omega Bio-Tek, Inc., USA) and further purified with the RNeasy MinElute Cleanup Kit (Qiagen). RNA purity was determined using the 260/280 nm absorbance ratio (NanoDrop ND-1000). Polymerase chain reaction (PCR) primers were designed based on complementary deoxyribonucleic acid (cDNA) sequences from the NCBI Sequence database. The PCR primers used in this study were shown as following: Postn, Forward 5'CAACGCAGCGCTATTCTGAC-3' and Reverse 5'-CCAAGTTGTCCCAAGCCTCA-3'; GAPDH, Forward 5'-CAGCCGCATCTTCTTGTGC-3' and Reverse 5'-GGTAACCAGGCGTCCGATA-3'. Quantitative
SYBR-Green real-time PCR was used for five independent RNA samples from each treatment group for each time point. Each reaction was repeated three times. The reaction was subjected to a 45 -cycle amplification at $95^{\circ} \mathrm{C}$ for 15 seconds, at $95^{\circ} \mathrm{C}$ for 5 seconds, and at $60^{\circ} \mathrm{C}$ for 30 seconds. Relative gene expression levels were calculated using the 22DDCt method. Target mRNA levels were normalized to glyceraldehyde-phosphate dehydrogenase (GAPDH) mRNA. Differences between E-group and S-group were analyzed using analysis of Student's unpaired t-tests. $\mathrm{P}<0.05$ was considered statistically significant. Statistical analyses were performed using SPSS version 19.0 (SPSS Inc.).

\subsection{Immunohistochemistry}

$5-\mu \mathrm{m}$ sections from the medial femoral condyle of each joint were following the procedure. After deparaffinization and hydration, antigen retrieval using $0.01 \mathrm{M}$ citrate butter and microwaving for 20 minutes. Then slides were cooled in room temperature. Washing of sections in phosphate buffered saline (PBS) solution. Quenching of endogenous peroxidase activity with $3 \% \mathrm{H}_{2} \mathrm{O}_{2}$ in methanol for 15-20minutes. Washed slides with PBS for 3 times. Blocked with $5 \%$ goat serum for 1 hour, and the incubated with antiperiostin polyclonal antibody (Abcam, Cambridge, MA) at a dilution of 1:50 and a negative control with PBS at $4{ }^{\circ} \mathrm{C}$ overnight. Next day, after washed in PBS for 3 times, incubated with peroxidase-conjugated secondary antibodies of dilution $1: 200$ at $37^{\circ} \mathrm{C}$ for 1 hour, then washed in PBS for 3 times. Diaminobenzidine substrate (DAKO, USA) colorimetric detection was carried out for equal time periods for each section followed by Mayer's hematoxylin (Sigma Corp., St. Louis, MO) counterstaining. Sections used for experiments for each protein were obtained from 5 different animals, with reproducible results. Images were captured by Leica DMI 3000 B microscope (Germany) and software (Leica Application Suite V3).

\section{Result}

\subsection{Real-Time PCR}

Total RNA was extracted from the cartilage and assayed by real-time RT-PCR. We found that the mRNA levels of Postn of chondrocytes in E-group were significantly higher than that in S-group at 1,2 and 4 weeks post-surgery. Interestingly, up-regulation of Postn expression of the cartilage appeared to increase sequentially and in a time-dependent manner with disease progression (Figure 1). As showed in the Table 1, the expression of Postn was about 2-fold higher at 1 week $(\mathrm{P}=0.08<0.05), 4$-fold higher at 2 weeks $(\mathrm{P}=0.003<0.05)$ and 4 -fold higher at 4 weeks $(\mathrm{P}=0.034<0.05)$ post-surgery in E-group compared to S-group. 


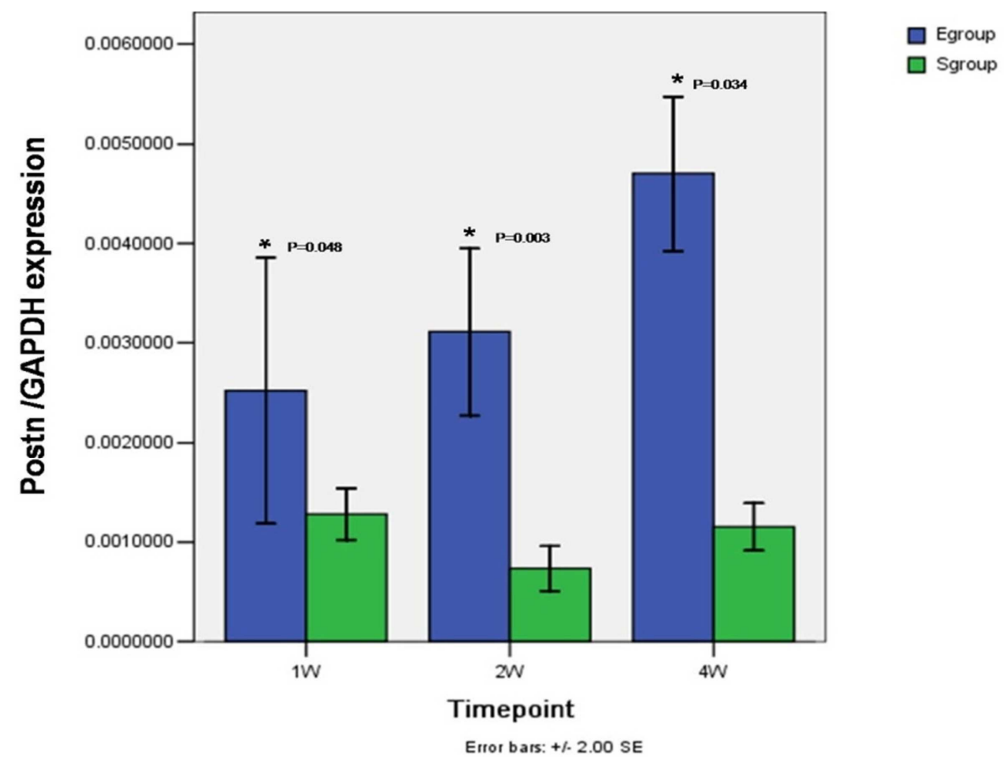

Figure 1. mRNA levels of Postn of chondrocytes in E-group were significantly higher than that in S-group at 1, 2 and 4 weeks post-surgery. The values are the mean and SEM of the gene expression levels in 5 animals, as determined by $\triangle C$ t analysis, normalized to GAPDH expression, and relative to the expression levels of S-group. ${ }^{*} p<0.05$ ( E-group versus S-group).

\subsection{Validation of Postn Expression at the Protein Level}

Immunohistochemistry staining was performed to examine the protein levels of Postn in tissue sections from the E-Group and the S-Group. As showed in the Figure 2, the cartilage cells mainly expressed Postn in their cytoplasm, and stronger positive signals in cartilage cells indicates Postn protein expression levels were markedly increased in the E-Group compared with the S-Group at 1, 2 and 4 weeks post-surgery. However, cartilage cells of the S-Group appeared negative at all three time points post-surgery. Interestingly, positive signal for Postn of the E-Group increase in a time-dependent manner with disease progression, which was in complete agreement with the expression levels of this gene determined by real-time PCR. Specially, intensive positive cartilage cells mainly located in the superficial zone of articular cartilage, however, in the deeper zone of cartilage, the positive staining was rarely detected, supporting our hypothesis that Postn expression induced by pathologic overload forces on cartilage.

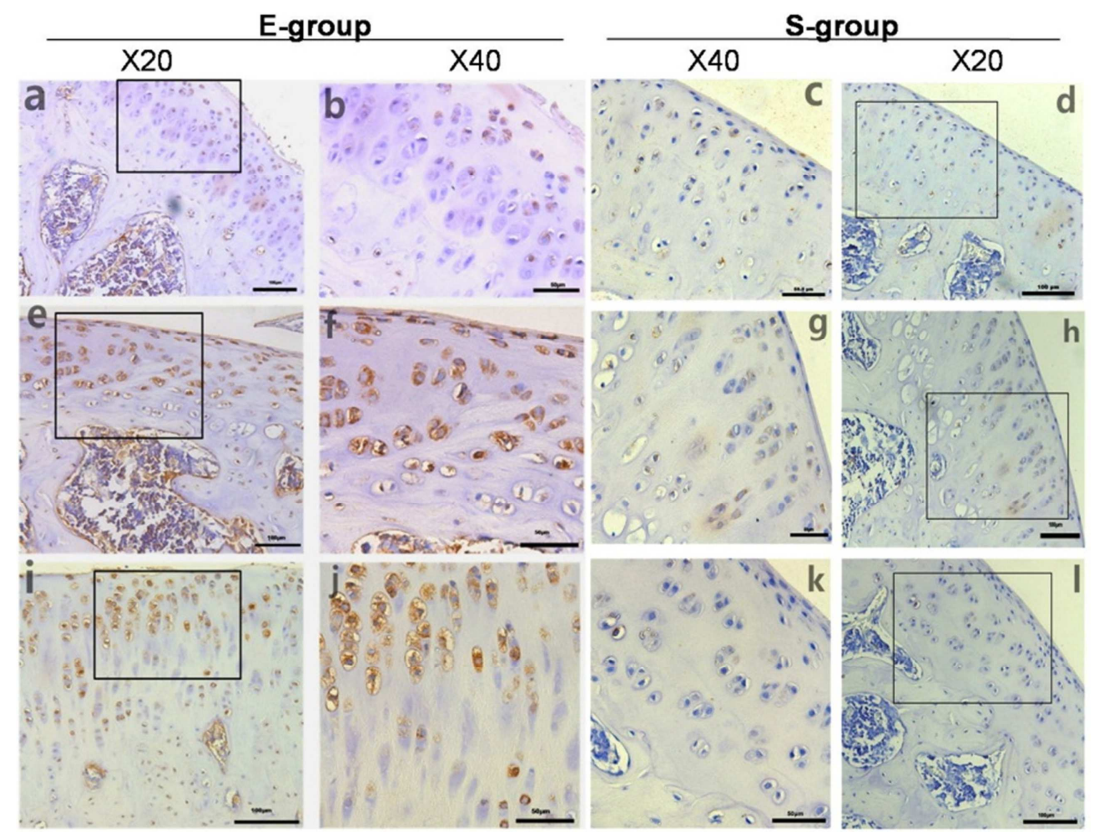

Figure 2. Evaluation of the expression levels of Postn in the cartilage of the E-group and the S-group using immunohistochemistry staining. The antibody against Postn was used to assess the spatial and temporal expression of the protein by colorimetric detection (brown precipitate). As showed in the Figure, the cartilage cells mainly expressed Postn in their cytoplasm, and stronger positive signals in cartilage cells indicates Postn protein expression levels were markedly increased in the E-Group compared with the S-Group at 1, 2 and 4 weeks post-surgery. Cartilage cells of the S-Group appeared negative at all three time points post-surgery. Intensive positive cartilage cells mainly located in the superficial zone of articular cartilage. Positive staining was rarely detected in the deeper zone of cartilage. All sections were counterstained with hematoxylin (blue stain). The scale bars for $20 X$ and $40 X$ are $100 \mu m$ and $50 \mu m$ respectively. 


\section{Discussion}

Since it is currently impossible to obtain adequate samples from humans to study the initiation and early stages of OA, using animal models is a necessary method to study the pathogenic mechanisms of OA at very early stages in vivo. Recent advances in animal OA model have allowed us to quantify molecular changes of OA over time $[8,9]$. Postn is a vitamin K-dependent glutamate-containing matri-cellular protein, originally isolated from a mouse osteoblast cell [5]. As an extra-cellular matrix (ECM) protein, Postn involved in regulating intercellular adhesion via an interaction with other ECM proteins [10]. More recently, several studies revealed that Postn may be responsible for the pathogenesis of OA. Ryota et al. reported that Postn was upregulated mainly in the chondrocytes near the erosive area and less immunoreactivity in deeper zones in human OA cartilage [11]. Mukundan Attur et al. also reported that high Postn levels in human and rodent OA cartilage, further study revealed that Postn significantly enhanced expression of MMP-13 and ADAMTS4 and promoted cartilage degeneration through collagen and proteoglycan degradation [12]. Analysis of osteoarthritic plasma and synovial fluid demonstrated that Postn levels was significantly higher and were positively correlated with the radiographic severity of knee OA [13]. J. C. Rousseau et al. also reported that serum Postn is associated with prevalence and the risk of progression of knee OA in women [14]. Taken together, the previous studies demonstrated that Postn may play prominent roles in pathogenic mechanisms of OA.

It is believed that mechanical stress is the major etiology in OA $[15,16]$. Postn expression is maintained in certain adult tissues including the periosteum and periodontal ligament. Postn deletion results in impairment of the ability of the periodontal ligament in response to mechanical stresses [6]. Our previous study in experimental rat OA model found that the Postn gene is significantly upregulated in the subchondral bone at 1 week post-surgery, but was not differentially expressed at 2 and 4 weeks post-surgery [7]. However, in the current study, Postn expression levels were markedly increased in the cartilage at 1,2 and 4 weeks post-surgery. Although the mechanism that induced the difference of expression of Postn between between subchondral bone and cartilage remained unknown, different mechanical force on the two tissues may involve in $[16,17]$. Specially, up-regulated Postn cartilage cells mainly located in the superficial zone of articular cartilage, however, in the deeper zone of cartilage, the positive staining was rarely detected, supporting our hypothesis that Postn expression induced by pathologic forces on cartilage. In the OA model used in this study, knee joint surgical instability induced pathologic overload forces. As we known, the surface layer of cartilage is exposed to higher pathologic pressure and shear stress than deeper layer of cartilage and the subchrodral bone $[17,18]$. Additionally, Postn is a secreted protein synthesized by osteoblasts and chondrocytes and it could participate to the crosstalk between subchondral bone and cartilage and it may involve different signaling pathways in different tissue [14].
More research needs to focus on the mechanism by which Postn in different tissue responds to mechanical forces, how it initiates these reactions, and how it relates to OA.

The main objective of this study was to analysis the Postn expression in cartilage in multi-stages of an experimental rat OA model. In the present study, the time course of the expression changes of Postn were revealed in cartilage in very early stages of rat experimental OA using real-time PCR and immunohistochemistry analysis. Consistent with pervious results, this study will be of benefit to those subsequent studies using the OA model to evaluate the outcomes of Postn epxpression in mechanism of $\mathrm{OA}$.

\section{Conclusion}

Postn may play prominent roles in pathogenic mechanisms of OA and may be a perfect therapeutic target for blocking the progress of osteoarthritis at a very early stage, when the cartilage is still intact.

\section{Acknowledgements}

This work was supported by grants obtained from the China Scholarship Foundation (Grant No. 201506385051), the Natural Science Foundation of Guangdong Province, China (Grant No. 2015A030310240), the Medical Research Foundation of Guangdong Province, China (Grant No. A2014261), and the Scientific and Technological Projects of Zhuhai City, Guangdong Province, China (Grant No. 2013D0401990009 and No.20161027E030050).

\section{Competing Interests}

The authors declare that they have no competing interests.

\section{References}

[1] Steinberg J, Zeggini E: Functional genomics in osteoarthritis: Past, present and future. J Orthop Res 2016; 13: [Epub ahead of print].

[2] Varady NH, Grodzinsky AJ: Osteoarthritis year in review 2015: mechanics. Osteoarthritis Cartilage 2016; 24:27-35.

[3] Moore AC, Burris DL: Tribological and material properties for cartilage of and throughout the bovine stifle: support for the altered joint kinematics hypothesis of osteoarthritis. Osteoarthritis Cartilage 2015; 23:161-9.

[4] Wang $\mathrm{H}$, Chen $\mathrm{T}$, Torzilli $\mathrm{P}$ et al: Dynamic contact stress patterns on the tibial plateaus during simulated gait: a novel application of normalized cross correlation. J Biomech 2014; 47:568-74.

[5] Horiuchi K, Amizuka N, Takeshita S et al: Identification and characterization of a novel protein, periostin, with restricted expression to periosteum and periodontal ligament and increased expression by transforming growth factor beta. J. Bone Miner. Res 1999; 14: 1239-1249. 
[6] Rios H, Koushik SV, Wang H et al: periostin null mice exhibit dwarfism, incisor enamel defects, and an early-onset periodontal disease-like phenotype. Mol Cell Biol 2005; 25:11131-44.

[7] Zhang R, Fang H, Chen Y et al: Gene Expression Analyses of Subchondral Bone in Early Experimental Osteoarthritis by Microarray. PLoS One 2012; 7: e32356.

[8] Yucel I, Karaca E, Ozturan K, et al: Biomechanical and histological effects of intra-articular hyaluronic acid on anterior cruciate ligament in rats. Clin Biomech (Bristol, Avon) 2009; 24:571-6.

[9] Botter SM1, Glasson SS, Hopkins B, et al: ADAMTS5L/L mice have less subchondral bone changes after induction of osteoarthritis through surgical instability: implications for a link between cartilage and subchondral bone changes. Osteoarthritis Cartilage 2009; 17:636-45.

[10] Takayama G, Arima K, Kanaji $\mathrm{T}$ et al: Periostin: a novel component of subepithelial fibrosis of bronchial asthma downstream of IL-4 and IL-13 signals. Allergy Clin Immunol 2006; 118:98-104.

[11] Chijimatsu R, Kunugiza Y, Taniyama $\mathrm{Y}$ et al: Expression and pathological effects of periostin in human osteoarthritis cartilage. BMC Musculoskelet Disord 2015; 16:215.

[12] Attur M, Yang Q, Shimada K et al: Elevated expression of periostin in human osteoarthritic cartilage and its potential role in matrix degradation via matrix metalloproteinase-13. FASEB J 2015; 29:4107-21.

[13] Honsawek S, Wilairatana V, Udomsinprasert W et al: Association of plasma and synovial fluid periostin with radiographic knee osteoarthritis: Cross-sectional study. Joint Bone Spine 2015; 82:352-5.

[14] Rousseau JC, Sornay-Rendu E, Bertholon C et al: Serum periostin is associated with prevalent knee osteoarthritis and disease incidence/progression in women: the OFELY study. Osteoarthritis Cartilage 2015; 23:1736-42.

[15] Buckwalter JA, Anderson DD, Brown TD et al: The roles of mechanical stresses in the pathogenesis of osteoarthritis: implications for treatment of joint injuries. Cartilage 2013; 4:286-94.

[16] Koike M, Nojiri H, Ozawa Y et al: Mechanical overloading causes mitochondrial superoxide and SOD2 imbalance in chondrocytes resulting in cartilage degeneration. Sci Rep. 2015; 5:11722.

[17] Madry H, van Dijk CN, Mueller-Gerbl M et al: The basic science of the subchondral bone. Knee Surg Sports Traumatol Arthrosc. 2010; 18:419-33.

[18] Lajeunesse D1, Reboul P et al: Subchondral bone in osteoarthritis: a biologic link with articular cartilage leading to abnormal remodeling. Curr Opin Rheumatol. 2003; 15:628-33. 